Projets

de paysage

\section{Projets de paysage}

Revue scientifique sur la conception et l'aménagement de l'espace

15 | 2016

L'observation et les observatoires de paysage

\title{
L'approche patrimoniale comme outil d'analyse et de mise en œuvre du projet de territoire en Italie
}

Le plan paysager de la Toscane

Using the Heritage as an Approach to Analysing the Implementation of

Development Planning in Italy - The Landscape Plan in Tuscany

\section{Maria Rita Gisotti}

\section{OpenEdition \\ Journals}

Édition électronique

URL : http://journals.openedition.org/paysage/6772

DOI : $10.4000 /$ paysage.6772

ISSN : 1969-6124

\section{Éditeur :}

École nationale supérieure du paysage de Versailles-Marseille, Institut national des sciences appliquées Centre Val de Loire - École de la nature et du paysage, École nationale supérieure d'architecture et de paysage de Bordeaux, École nationale supérieure d'architecture et de paysage de Lille, Agrocampus Angers

Référence électronique

Maria Rita Gisotti, « L'approche patrimoniale comme outil d'analyse et de mise en œuvre du projet de territoire en Italie », Projets de paysage [En ligne], 15 | 2016, mis en ligne le 31 décembre 2016, consulté le 13 juin 2020. URL : http://journals.openedition.org/paysage/6772 ; DOI : https://doi.org/10.4000/ paysage. 6772

Ce document a été généré automatiquement le 13 juin 2020

Projets de paysage 


\title{
L'approche patrimoniale comme outil d'analyse et de mise en œuvre $\mathrm{du}$ projet de territoire en Italie
}

\author{
Le plan paysager de la Toscane \\ Using the Heritage as an Approach to Analysing the Implementation of \\ Development Planning in Italy - The Landscape Plan in Tuscany
}

Maria Rita Gisotti

Depuis plusieurs années en Italie s'est développée une vision structurelle et patrimoniale du territoire et du paysage comme réceptacles de valeurs stratifiées par le processus ambiant de coévolution homme-milieu. Dernièrement, cette approche s'est concrétisée dans certaines expériences de planification du paysage. Les plus mûres ont abouti à l'approbation du plan, alors que les autres ont coïncidé avec des plans adoptés ou en cours d'élaboration. Le projet de territoire qui découle de cette vision réactualise certaines règles implicites au sein du patrimoine territorial, toujours valables pour leur rationalité métahistorique.

2 Cette contribution a pour but d'illustrer les fondements de l'approche patrimoniale et du plan paysager de la Toscane en tant qu'application concrète. L'article définit d'abord le contexte juridique et scientifique dans lequel s'ancre ce plan, puis illustre synthétiquement les principaux mots-clés de l'interprétation patrimoniale, pour décrire enfin la structure et les paradigmes du plan toscan. En conclusion, il est proposé une réflexion sur les passages et les instruments qui peuvent supporter la nouvelle conception de préservation du paysage véhiculée ici. L'importance de cette expérience réside dans le fait qu'elle constitue un banc d'essai efficace - et presque inédit - pour tester l'application de l'approche patrimoniale à un projet de territoire d'échelle régionale. 


\section{Le cadre normatif et scientifique}

Depuis 2004 en Italie, suite à l'approbation du code des biens culturels et du paysage, les régions sont chargées d'élaborer leur plan paysager, conçu comme un outil d'analyse et d'aménagement non seulement des espaces remarquables, mais aussi de l'ensemble du territoire régional ${ }^{1}$. Il s'agit d'une avancée significative dans le milieu italien de la préservation du paysage, où les plans paysagers existent depuis 1985. Cependant, très peu de régions en ont élaboré 2 . Pendant longtemps, en revanche, a persisté une sorte de "double régime ", avec d'un côté les territoires inscrits et classés au titre des paysages soumis à des formes de protection strictes, et de l'autre un contexte régional souvent dépourvu de règles de gestion des transformations. Le code des biens culturels et du paysage, en introduisant l'obligation pour les régions d'élaborer un plan paysager pour l'ensemble de leur territoire et non seulement pour les biens inscrits et classés, transpose implicitement les avancées de la Convention européenne du paysage (CEP). Celle-ci, comme on le sait, "s'applique à tout le territoire des parties et porte sur les espaces naturels, ruraux, urbains et périurbains. Elle inclut les espaces terrestres, les eaux intérieures et maritimes. Elle concerne tant les paysages pouvant être considérés comme remarquables, que les paysages du quotidien et les paysages dégradés ${ }^{3}$ ». La définition de paysage formulée par le code italien reprend celle de la CEP, en affirmant que le paysage est un territoire "dont le caractère résulte de l'action de facteurs naturels, humains et de leurs interrelations ${ }^{4} »$.

Il s'agit d'une conception structurelle de paysage qui, en Italie, est enracinée dans la tradition d'études historico-géographiques représentée par Emilio Sereni et Lucio Gambi $^{5}$ - liée à celle française de Marc Bloch et Fernand Braudel (Scazzosi, 2011) - et qui a été poursuivie dans de nombreuses expériences pratiques et réflexions théoriques (Di Pietro, 2004; Gambino, 2010; Poli, 2011). Le paradigme structurel lit le paysage comme résultat de l'interaction d'une multiplicité de facteurs, qui se sont succédé "tout au long de la longue durée historique»: donc non comme un «champ opérationnel quasiment abstrait mais comme produit d'une très longue et très lente stratification qu'on doit connaître pour pouvoir intervenir", "un palimpseste complexe qu'il faut comprendre et déchiffrer » (Corboz, 1985, p. 27, trad. de l'auteur) ${ }^{6}$. $\mathrm{Au}$ sein de cette interprétation structurelle se niche une approche patrimoniale et territorialiste, bien représentée (entre autres) par les deux plans paysagers aujourd'hui approuvés selon la réglementation prévue par le code des biens culturels et du paysage : les plans paysagers des Pouilles et de la Toscane ${ }^{7}$. Selon ce type d'approche, le paysage peut être analysé comme patrimoine en tant que dépôt de valeurs, tant matérielles qu'immatérielles (Magnaghi, 2000 ; Gambino, 2009 ; Marson, 2013 ; Gisotti, 2015).

\section{La vision territorialiste et patrimoniale : les coordonnées principales}

Cette approche s'est confirmée en Italie à partir des années 1990 avec les études d'Alberto Magnaghi et son idée de "développement local autosoutenable ", systématisée dans son ouvrage Il progetto locale $(2000)^{8}$. Autour et à partir de cette idée, beaucoup d'études ont été conduites dans les années suivantes - des recherches universitaires mais surtout des recherches-actions systématiquement liées à des 
parcours de formation ${ }^{9}$ - qui ont constitué une école de pensée appuyée sur certains concepts de base ${ }^{10}$.

\section{Territoire comme produit de coévolution}

6 Le paradigme fondamental est une conception nouvelle du territoire par rapport à l'urbanisme traditionnel, qui notamment le traitait comme une tabula rasa, une « feuille blanche » support des activités anthropiques. Au contraire, le territoire est ici conçu comme un organisme doté d'une "personnalité ${ }^{11}$ ", "un sujet, un organisme vivant complexe, produit de la rencontre entre événements culturels et naturels, composé de lieux (ou de régions) dotés d'identité, d'histoire, d'un caractère et d'une structure de longue durée » (Magnaghi, 2014, p. 10). Le territoire n'existe donc pas par nature, comme une longue tradition d'études de géographie historique l'a montré. Il n'est pas une simple aire géographique, ni un environnement totalement intact. Au contraire, il est le produit du travail de domestication porté par l'homme sur son milieu, dans un processus de coévolution: depuis des milliers d'années, homme et milieu ont évolué ensemble. Le premier a accepté les limites imposées par la nature afin de préserver sa sécurité et survivre. Depuis les années 1950 et 1960, dans des contextes comme celui de l'Italie, pays resté jusque-là fortement rural, l'homme a interrompu ce parcours de coévolution, en développant de nouvelles technologies - relatives à l'implantation d'infrastructures et à l'exploitation des ressources - qui lui ont permis de dépasser ces limites. L'arrêt du processus de «territorialisation » (Turco, 1984; Magnaghi, 2000) a souvent conduit à des catastrophes «naturelles » (surtout inondations et glissements de terrain).

7 La territorialisation coévolutive a engendré de nombreuses strates, c'est-à-dire des sédiments qui peuvent être matériels (rues, bâtiments, villes, cultures agricoles) mais aussi immatériels et cognitifs. Il s'agit surtout de modes de fonctionnement, par exemple une relation vertueuse entre les caractères du sol (du point de vue de la pente, de la composition géomorphologique, de l'exposition au soleil) et son usage par l'homme: en généralisant, on peut dire qu'aux deux extrémités de la gamme des possibles utilisations anthropiques d'une zone, jusqu'aux années 1960, dans l'Italie collinaire, les humains ont privilégié, pour implanter leurs habitations, les meilleurs terrains au détriment des territoires trop raides, rocheux, mal ou peu ensoleillés, qu'ils ont destinés aux bois.

\section{Le patrimoine territorial et paysager}

8 La territorialisation a donc produit plusieurs « couches » sous forme d'objets physiques mais aussi de modes de bonne relation entre homme et milieu ambiant, lisibles finalement comme des règles structurelles. Dans la Toscane des collines, par exemple, la structure profonde des implantations humaines est ordonnée par la « règle de crête ", d'ailleurs depuis peu négligée. Le respect de cette règle permettait de bâtir sur les points stables et sûrs, et en même temps de garder les terrains en pente pour les cultures. Des terrasses et des murs en pierre sèche soutenaient les sols plus raides, tout en permettant de les cultiver, et assuraient en même temps l'écoulement des eaux et la maitrise de l'érosion. La chaleur des murs ensoleillés améliorait la qualité de certaines cultures, comme la vigne et, par conséquent celle du produit final. 
Cette structure profonde a résisté aux transformations historiques grâce à sa rationalité métahistorique (Antrop, 2005 ; Baldeschi, 2002). Cela ne veut pas dire que les territoires et le paysage n'ont pas changé sur la durée. Au contraire : les niveaux plus superficiels (notamment les cultures) ont été très fréquemment remplacés, et même à l'intérieur de la structure profonde - formée par le relief, le réseau hydrologique, le système d'implantations et celui des ouvrages de soutien des pentes -, le flux des modifications a été continu, mais ces dernières étaient toujours guidées par le respect de règles comme celle de «l'implantation de crête ».

10 L'idée du patrimoine territorial découle de cette interprétation structurelle. Le patrimoine peut en effet être conceptualisé comme "ensemble des structures de longue durée produites par la coévolution entre milieu naturel et implantations humaines ${ }^{12} »$. Sa valeur n'est pas simplement testimoniale. Elle est surtout de type fonctionnel parce qu'il contient des principes et des configurations toujours viables et opérationnels, et donc réactualisables. Le patrimoine territorial, a écrit Roberto Gambino, n'est pas à entendre comme « un gisement inerte de "choses" hétérogènes et sans lien, d'où extraire ce qui est nécessaire [mais comme] un système plus ou moins cohérent et interconnecté d'héritage historique, culturel et naturel, tangible et intangible, d'appartenance à un réseau de relations qui lient lieux et formations sociales [...]. C'est ici que l'analyse peut découvrir les "racines du futur" [...] avec qui construire les plans territoriaux et les projets de territoire » (Gambino, 2010, cité dans Gisotti, 2015, p. 48) $)^{13}$.

\section{La biorégion urbaine}

11 Conceptualiser le territoire comme produit historique de coévolution entre homme et milieu ambiant - une coévolution qui a sédimenté des valeurs patrimoniales matérielles et immatérielles - a impliqué une révision radicale du modèle de développement actuel, à la base de pauvreté économique, environnementale, sociale, culturelle. La biorégion urbaine représente un possible scénario de développement local autosoutenable pour la reconstitution d'une relation coévolutive vertueuse entre homme et milieu $^{14}$. Dans la pensée territorialiste, elle a été définie comme "ensemble de systèmes territoriaux locaux fortement transformés par l'homme, caractérisés par la présence d'une pluralité de centres urbains et ruraux organisés en systèmes réticulaires et non hiérarchisés. Ces systèmes sont reliés entre eux par des rapports environnementaux qui tendent à réaliser un bouclage des cycles de l'eau, des déchets, de l'alimentation et de l'énergie » (Magnaghi, 2014, p. 82). Coïncidant à la fois avec un bassin hydrographique, un territoire provincial, une unité paysagère, la biorégion urbaine peut avoir une dimension extrêmement variable. Son attribut fondamental est de matérialiser des relations positives entre villes et établissements d'un côté et leurs systèmes agroforestiers de l'autre, reliés par un échange mutuel.

12 Les espaces ouverts de la biorégion urbaine sont à cette fin des instruments stratégiques ${ }^{15}$ : espaces naturels et agricoles entremêlés aux urbanisations contemporaines, ils représentent des dépôts de valeurs patrimoniales pour leur capacité à proposer une multiplicité de services écosystémiques (Costanza et al., 1997; Millennium Ecosystem Assessment, 2005 ; TEEB 2012), la plupart liés à une agriculture multifonctionnelle (Donadieu 2006; Fanfani 2009; Magnaghi, Fanfani 2010; Mininni, 2013). En particulier, ils agissent au niveau des équilibres hydrogéomorphologiques et 
écologiques, des systèmes productifs locaux, de la production alimentaire, de la recomposition morphologique et paysagère, de la création et récupération de l'espace public à l'échelle urbaine, métropolitaine et territoriale.

Figure 1. Scénario biorégional pour la Toscane centrale

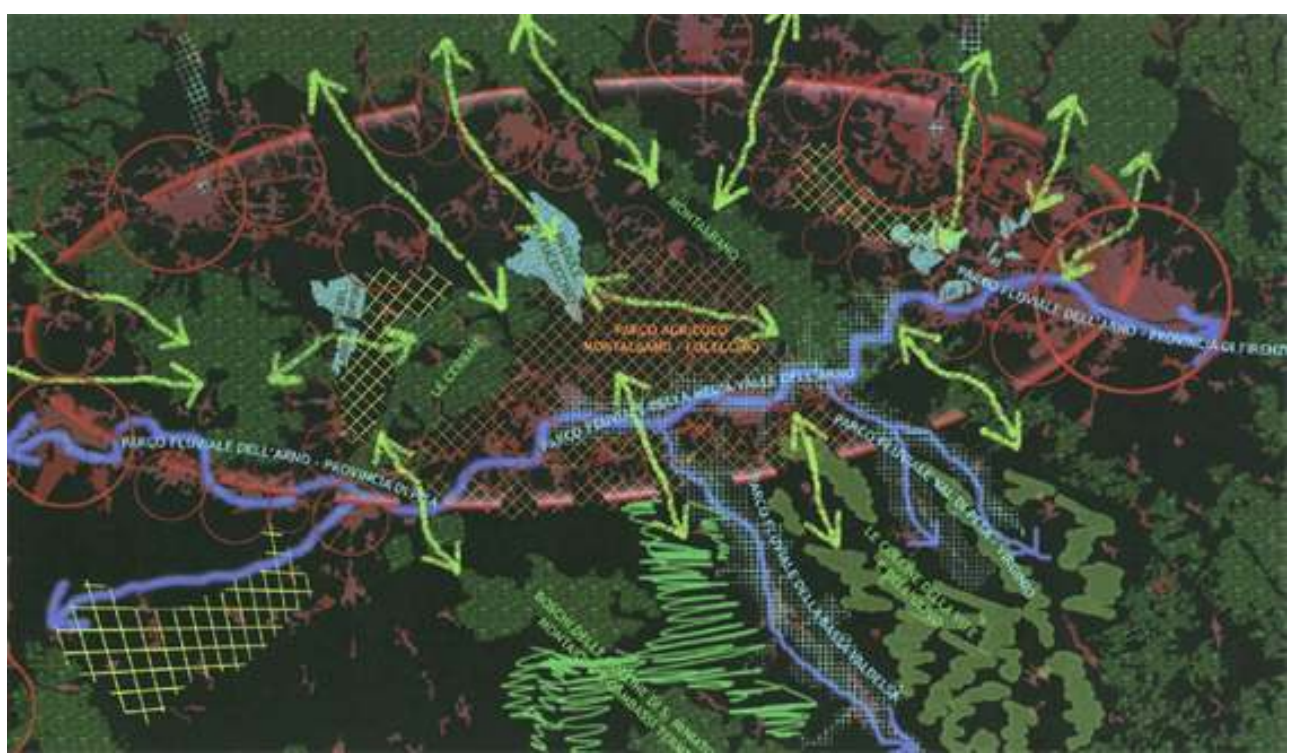

Sources : Magnaghi, Fanfani, 2010.

\section{L'expérience du plan paysager de la Toscane}

Comment opère-t-on le passage de la théorie territorialiste et patrimoniale à la pratique d'aménagement sur un territoire ? Durant les vingt dernières années, l'Italie a connu plusieurs expérimentations à l'échelle communale ou provinciale. Dernièrement, une avancée significative fut la rédaction des plans paysagers, à savoir des plans territoriaux que les régions ont l'obligation d'élaborer depuis 2004, et qui ont une valeur normative supérieure à tous les autres outils d'urbanisme et d'aménagement, tant réglementaires que sectoriels. La Toscane est, avec les Pouilles, la région qui a le plus avancé dans cette démarche ${ }^{16}$. Le plan a été réalisé avec la collaboration du Centre interuniversitaire de sciences du territoire (CIST), un organisme académique qui regroupe des spécialistes de nombreuses disciplines autour du projet de développement d'un système intégré de connaissance sur les sciences du territoire. 
Figure 2. Une image de la géographie physique de la Toscane (1:250.000)

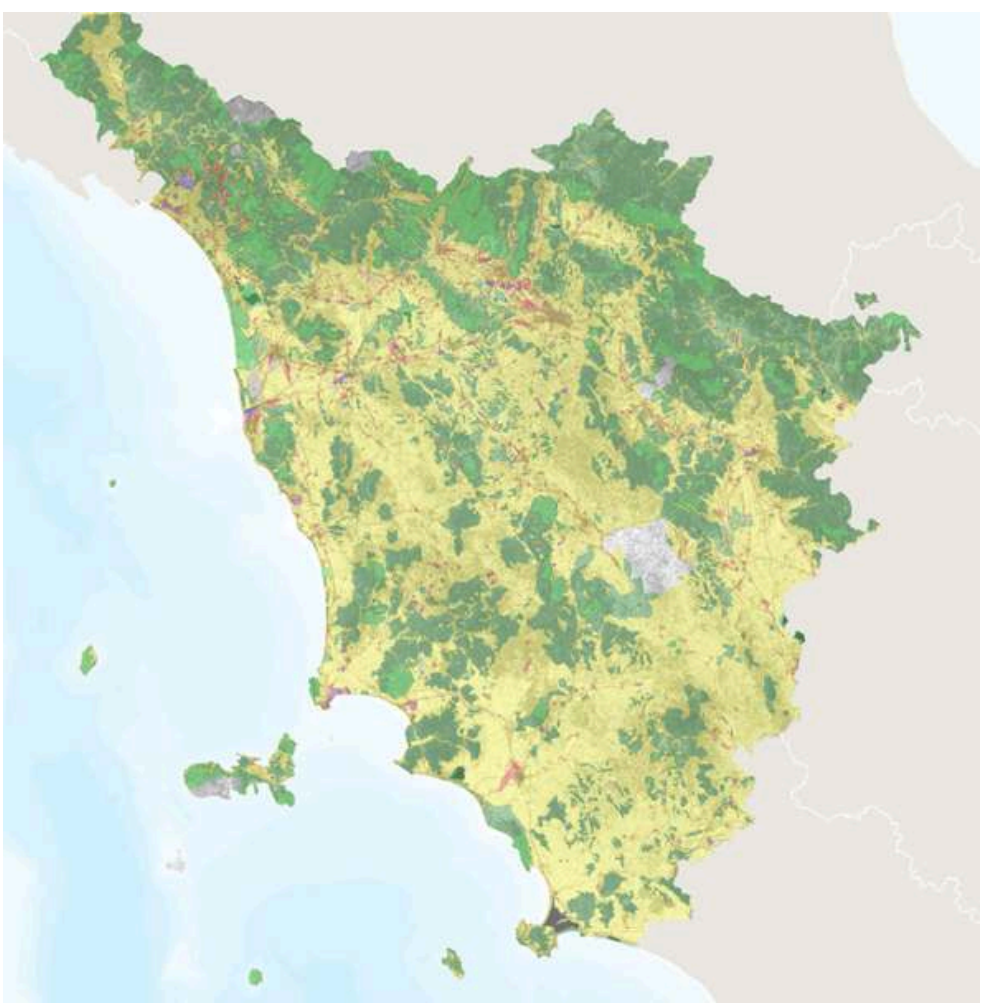

Le but du plan est de poursuivre la promotion et la réalisation d'un développement socioéconomique durable et d'un usage conscient du territoire régional. Le pivot et instrument essentiel de cette stratégie est le patrimoine territorial et paysager, conçu comme «l'ensemble des structures de longue durée produit par la coévolution entre milieu naturel et installations humaines, dont la valeur est reconnue pour les générations présentes et futures ${ }^{17}$ "(Marson, 2016). Le patrimoine, «bien commun dont on doit préserver les conditions de reproduction, la viabilité des usages et la durabilitée ${ }^{18}$ ", représente une valeur ajoutée extraordinaire pour la Toscane, non seulement en matière d'identité et d'attractivité exogènes, mais aussi pour sa multifonctionnalité et comme dépôt de ressources pour la production de richesses endogènes. Poser le patrimoine comme fondement du plan comporte d'abord un effort de reconnaissance approfondie et détaillée du patrimoine même, dans toutes ses composantes. Ce choix implique aussi de décoder le "projet implicite» (Dematteis, 1995) du territoire, de comprendre et de décrire les éléments et les relations constitutifs du patrimoine et de les assumer comme règles pour la gestion des transformations futures. Diagnostic et projet territorial, état des lieux et scénarios possibles sont, en conséquence, effectivement étroitement liés.

La structure du plan paysager de la Toscane est assez complexe, avec de nombreuses élaborations et produits, qui se réfèrent aussi bien à l'échelle régionale qu'à celle de l'unité (ambito) de paysage. Cependant, en schématisant, on peut dire qu'à partir de la posture méthodologique précédemment illustrée, chaque unité paysagère a été traitée à deux niveaux : un diagnostic très poussé et une partie de projet qui codifie le cadre normatif ${ }^{19}$. Le diagnostic a été subdivisé en trois sections consacrées respectivement à la description interprétative, aux invariantes structurelles et aux interprétations de 
synthèse. En revanche, la partie conceptuelle comprend les orientations pour les politiques sectorielles et les normes pour l'urbanisme réglementaire.

Figure 3. Carte des caractères du paysage avec sa légende (PIT Toscane, détail)

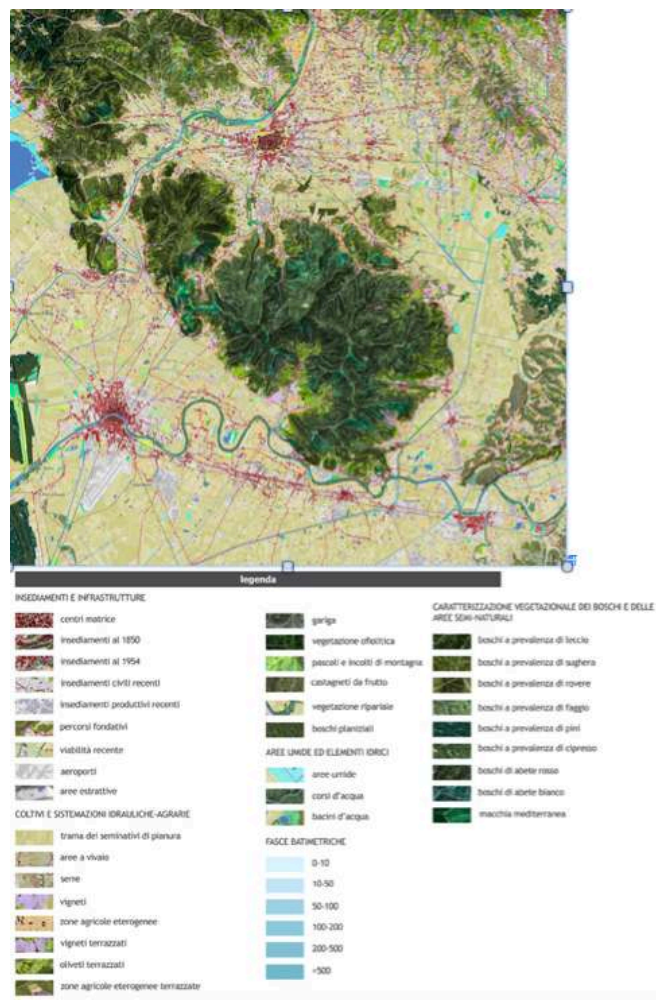

La carte a gagné la mention d'honneur dans la Reference Map Category of CaG/S Competition, 2014, organisée par la Cartography and Geographic Information Society (USA).

\section{Description interprétative et invariantes structurelles}

Le premier niveau du diagnostic vise à reconstruire le processus de structuration du paysage régional tout au long de l'histoire, tant d'un point de vue des aspects physiographiques, que de celui des réalisations anthropiques. En conséquence, les thématiques développées synthétisent les phases principales de la structuration géologique et géomorphologique du territoire, identifient les différents processus de territorialisation de la préhistoire à l'époque actuelle et les processus "d'artialisation » situés à l'origine de la reconnaissance du paysage. La prise en compte de dynamiques relatives à la biosphère, à la sociosphère et à la sémiosphere montre la portée de la stratification sur un paysage comme la Toscane, fortement anthropisé, et en même temps faisant l'objet de puissants flux d'esthétisation culturelle.

La deuxième partie du diagnostic mobilise un concept présent depuis longtemps dans le débat italien sur l'urbanisme et l'aménagement territorial, l'invariante structurelle (Maggio, 2014). En Toscane, ce terme fait son apparition dans la loi régionale d'aménagement du territoire $n^{\circ} 5$ du 1995, ensuite précisé dans celle $n^{\circ} 1$ du 2005, puis dans la loi $\mathrm{n}^{\circ} 65$ du 2014, strictement liée au plan paysager. La définition d'invariante structurelle donnée par la loi $n^{\circ} 65$ et par le plan paysager est en effet presque identique: "les invariantes structurelles identifient les caractères spécifiques, les principes génératifs et les règles pour définir les conditions de modification et de 
transformation du patrimoine territorial ${ }^{20} »$. Patrimoine et invariante sont donc deux termes réciproquement liés : le second permet d'identifier les caractères identitaires du premier dérivés de la longue durée, ainsi que les modalités de sa gestion comme « système vivant en constante mutation " (Magnaghi, 2016b). Il s'agit d'un passage très important qui soustrait le patrimoine à une interprétation déterministe et conservatrice qui le figerait à un état fixé, et le projette en revanche dans un flux de changement qui doit être non empêché mais géré.

Les invariantes structurelles se référent aux quatre principales composantes territoriales et paysagères: hydrogéomorphologiques, écosystémiques, des systèmes d'implantation et des paysages ruraux. Les corpus d'études relatifs à chaque invariante gardent une autonomie disciplinaire spécifique tout en maintenant des principes méthodologiques communs entre eux, à savoir :

- un système d'analyse appuyé sur la description des caractères structuraux, des dynamiques de transformation, des valeurs et des menaces;

- une approche morphotypologique destinée à identifier des « types » reconnaissables dans le territoire régional ${ }^{21}$;

- la représentation des aspects identitaires de chaque invariante dans une carte relative à l'ensemble du territoire régional.

Ce dernier passage constitue une innovation très significative en Italie : elle consacre le passage d'une connaissance analytique et d'une action réglementaire réservées seulement à des parties de valeur exceptionnelle (typiquement les biens inscrits et classés, les soi-disant vincoli), à une lecture détaillée et approfondie de tout le territoire régional, y compris les paysages dégradés. En outre, cette connaissance horizontale et homogène au niveau régional peut constituer une base pour des politiques d'aménagement cohérentes qui, surtout sur certaines thématiques (par exemple le risque hydrologique et géomorphologique), dépassent les limites administratives communales et les conflits qui en résultent. 
Figure 4. Carte et légende du réseau écologique de la $2^{\mathrm{e}}$ Invariante structurelle (PIT Toscane, détail)

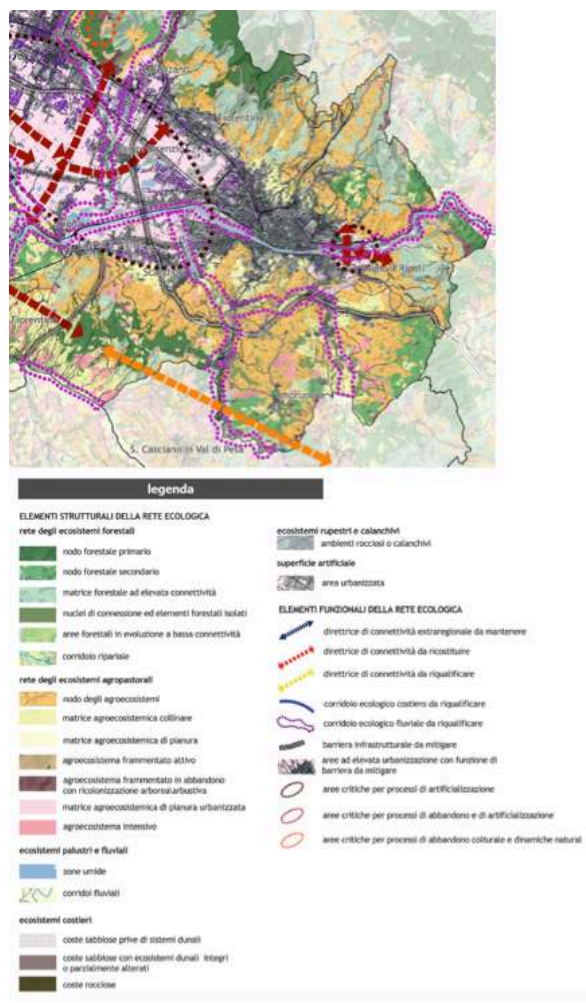

\section{Les interprétations de synthèse}

Dans l'analyse illustrée jusqu'à ce point, le paysage a été lu comme une stratification de couches qui se sont sédimentées tout au long de l'histoire. L'approche adoptée est fortement multidisciplinaire, en décomposant le paysage à travers l'analyse de ses caractères (hydrogéomorphologiques, écosystémiques, des systèmes d'implantation et des paysages ruraux). Au terme de ce parcours, il a été nécessaire de reconstituer les quatre grandes composantes paysagères (liées à autant d'invariantes structurelles) dans une représentation et une description synthétiques tel le patrimoine territorial et paysager, conçu comme sélection et intégration d'éléments et de structures de valeur identifiés dans l'étude de chaque invariante. Le rôle de ces structures - qui mêlent des éléments territoriaux à la fois historiques et contemporains - est multifonctionnel et proactif.

21 Considérons, à titre d'exemple, le rapport entre centres du système d'implantation historique et paysage rural dans les contextes de haute colline et de montagne. Cette relation donne lieu à des microcosmes isolés dans le bois, formés par l'ancien bourg et une mosaïque agricole tout autour. Ces terrains cultivés ont aujourd'hui une valeur importante à maints égards : non seulement de témoignage des systèmes agricoles traditionnels mais surtout en tant qu'éléments de discontinuité paysagère et de réserves de biodiversité. Ils coïncident, en effet, la plupart du temps avec des « nœuds du réseau écologique des agro-écosystèmes ${ }^{22}$ ", c'est-à-dire avec les High Nature Value Farmland (HNVF) identifiées par la stratégie UE pour la biodiversité 2020. En conséquence, même si les aires agricoles « ordinaires » mais avec valeur de « nœuds » ne bénéficient d'aucune forme de protection (comme par exemple les sites Natura 
2000), leur maintien mais avec valeur de "nœuds » est un objectif stratégique pour améliorer le niveau de perméabilité et de connectivité écologique de la région.

Figure 5. Carte du patrimoine territorial et paysager avec sa légende (PIT Toscane, détail)

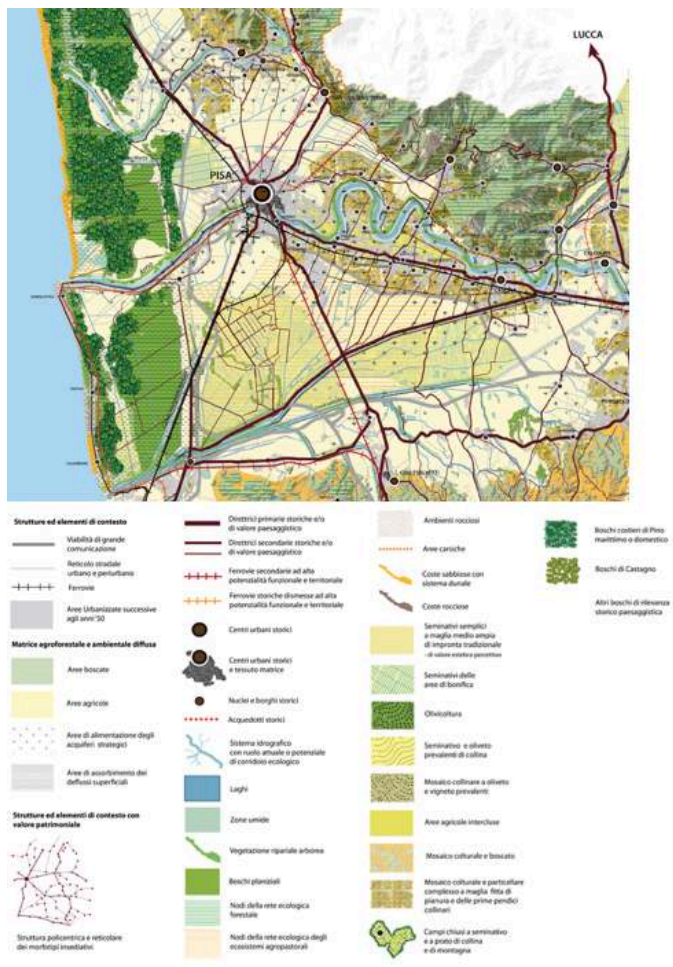

La lecture patrimoniale essaie de montrer les structures paysagères ayant une valeur tout en utilisant une représentation dense et identitaire visant à surmonter la traditionnelle inaccessibilité esthétique de la cartographie technique et à favoriser les modalités et formes de « production sociale » du paysage ${ }^{23}$. 
Figure 6. Carte des menaces avec sa légende (PIT Toscane, détail)

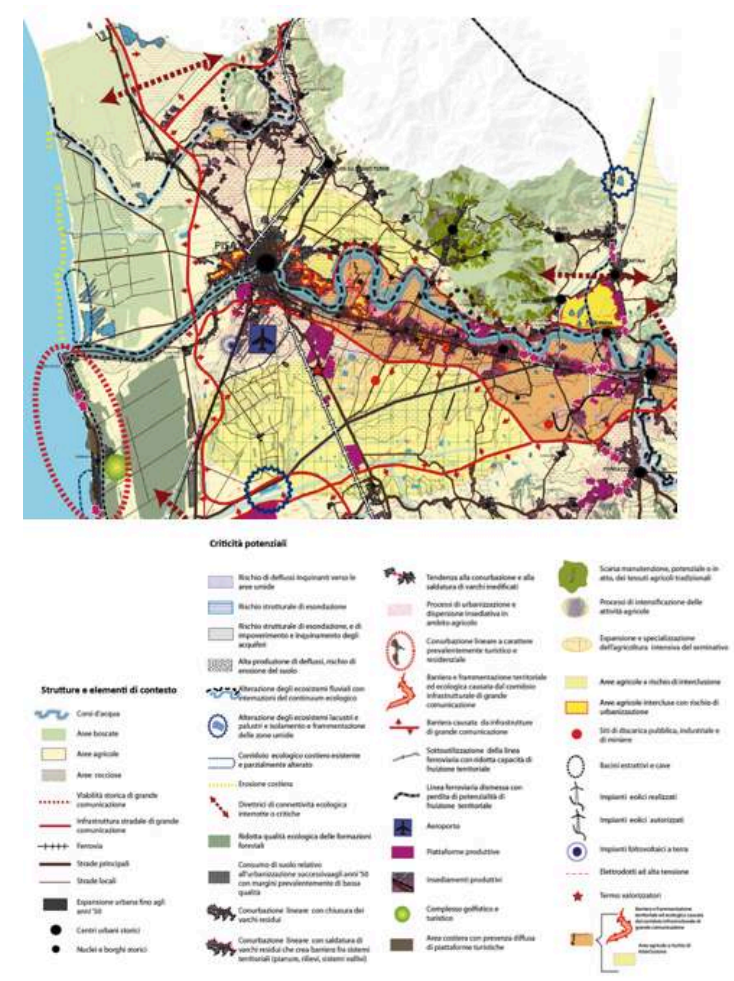

La carte fait partie des interprétations de synthèse et elle est conçue comme un ensemble d'éléments et de dynamiques qui affectent la structure du patrimoine paysager.

\section{Orientations et normes}

La section sur le diagnostic paysager illustré ci-dessus a abouti à une partie conceptuelle qui comprend des orientations pour les politiques sectorielles (par exemple infrastructures et mobilité, agriculture, environnement, déchets, tourisme, exploitation des mines, etc.) souvent liées aux fonds structurels européens, et des normes pour l'urbanisme réglementaire mises en œuvre essentiellement à travers les plans communaux. Les règles pour l'urbanisme sont formulées parmi l'énonciation d'un objectif paysager et d'un certain nombre de directives finalisées qui servent à son application, ces dernières éventuellement spécifiées par des lignes directrices conçues comme possibles modalités de leur mise en œuvre.

Les normes du plan paysager - pour des raisons liées d'abord à l'échelle du plan même ne s'appliquent pas à des interventions ou à des actions de détail mais définissent plutôt des principes-guides pour l'aménagement du territoire que tous les autres plans devront respecter. La nature des règles est fortement multidisciplinaire : elles dérivent en effet de la lecture croisée des éléments ayant valeur patrimoniale et des dynamiques critiques concernant les aspects hydrogéomorphologiques, écosystémiques, des systèmes d'implantation et des paysages ruraux. Elles reflètent en ce sens l'approche structurelle adoptée, et tendent à dépasser, même dans l'urbanisme réglementaire à échelle communale, une conception du paysage traditionnellement en faveur des aspects esthétiques et visuels. On peut dire que les normes essaient de traduire en 
règles d'aménagement les principes coévolutifs qui soutiennent la structure territoriale et paysagère toscane, et que le plan a reconnus comme valeurs patrimoniales.

Considérons à titre d'exemple l'un des objectifs pivot du plan, l'arrêt de la consommation de sol $^{24}$. Dans l'unité (ambito) Florence-Prato-Pistoia, cet objectif est la "préservation et [la] réhabilitation $d u$ caractère polycentrique du système d'implantation de la plaine ${ }^{25} »$. Il se traduit en une batterie de directives qui visent à :

- maintenir et réhabiliter les coupes d'urbanisation existantes, avec une attention particulière à celles situées le long des axes routiers pour leur valeur morphologique et écologique ;

- éviter de nouveaux processus d'étalement urbain, soigner les marges du bâti et sauvegarder les espaces ouverts non bâtis, surtout les terrains agricoles pour lesquels il est primordial de valoriser la multifonctionnalité ;

- préserver la lisibilité du système d'implantation historique en évitant de nouvelles expansions qui ne soient pas strictement nécessaires et en prévoyant, éventuellement, qu'elles soient cohérentes avec le contexte.

La tension conceptuelle et proactive qui caractérise les règles du plan paysager se concrétise aussi dans les "normes figurées ", illustrations des projets à activer pour la mise en œuvre du scenario envisagéér.

Figure 7. « Norme figurée » relative à l'ambito 06 Firenze-Prato-Pistoia (PIT Toscane)

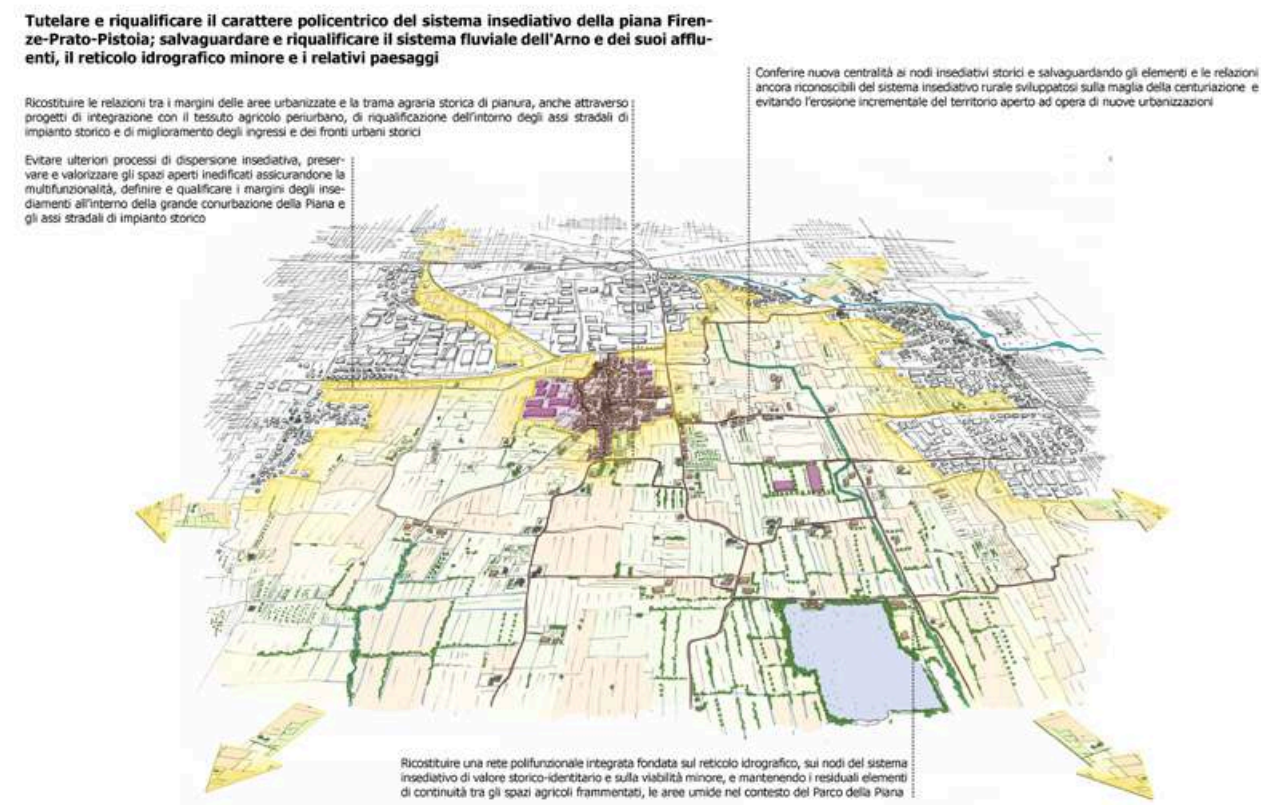

\section{Une réflexion conclusive}

27 L'interprétation structurelle dans laquelle s'inscrivent l'approche territorialiste et le plan paysager de la Toscane, qui en est une application, analyse le paysage comme le produit de plusieurs composantes. À partir de la reconnaissance de cette complexité, certains paysages régionaux peuvent être conçus comme patrimoines, c'est-à-dire comme systèmes interconnectés d'éléments et de relations multifonctionnelles et d'utilité collective. Les structures paysagères identifiées dans le plan ont en effet une 
valeur de type testimonial, culturel, esthétique mais en même temps fonctionnel et économique parce qu'elles recèlent une multiplicité de prestations (de la maîtrise du risque hydrologique et géomorphologique à l'amélioration de la biodiversité et de la connectivité écologique) qui se traduisent en bénéfices pour la collectivité. En s'appuyant sur cette vision, le plan a élaboré un cadre de diagnostic vaste et approfondi qui n'a pas simplement un rôle d'analyse, mais aussi de projet implicite dans la stratification territoriale.

Une telle vision de paysage, liant patrimoine et prestations collectives, peut créer une nouvelle manière de concevoir la préservation paysagère, au-delà de l'approche statique qui vise à congeler ou à muséifier le territoire en fonction d'un état donné, et identifier des principes et règles pour la gestion des transformations. Cela veut dire, par exemple, qu'à propos d'un paysage rural historique, on peut choisir de substituer les anciens ouvrages en pierre sèche si le coût de leur entretien est insoutenable, tout en gardant le même service de maîtrise de l'érosion avec des artefacts nouveaux qui soient cohérents avec le contexte paysager. L'outil méthodologique qui apparait le plus efficace pour l'application de cette approche est l'invariante structurelle, à la fois mode de fonctionnement du territoire et règle coévolutive pour son aménagement.

Pour que ce passage d'une préservation statique à une dynamique s'accomplisse, il est nécessaire de continuer à travailler au moins sur trois dimensions :

- l'affirmation d'une conception multidisciplinaire et intégrée du paysage, essentielle pour renverser le parcours traditionnel de la planification et de l'urbanisme selon lequel le projet de territoire régional découle de la somme (parfois incohérente) de plans de secteur ;

- la diffusion d'une modalité de représentation expressive et communicative des caractères patrimoniaux du paysage. Ce genre de cartes, sans perdre la fiabilité de l'information géographique, permet de rendre plus lisibles les aspects identitaires, de préfigurer des directions de projet, de soutenir le dialogue avec les utilisateurs non techniciens et, en général, avec les citoyens ;

- recueillir l'adhésion de la société locale à la mise en œuvre du projet de territoire envisagé. Un rôle de premier plan sera donc celui des collectivités territoriales et des communes qui sont tenues de conformer leurs outils d'aménagement et d'urbanisme au plan paysager.

Construire (à partir du début du plan et non a posteriori) une intersectorialité effective appuyée sur la lecture multidisciplinaire du paysage, promouvoir la construction sociale du projet paysager, diffuser une narration positive du plan et de ses effets de développement durable et en même temps assurer le fonctionnement de structures techniques de support pour la rédaction des plans communaux représentent des pivots pour la réalisation d'un scénario plaçant le patrimoine paysager telle « charpente de l'innovation ».

\section{BIBLIOGRAPHIE}

Antrop, M., "Why landscapes of the past are important for the future », Landscape \& Urban

Planning, $\mathrm{n}^{\circ} 70,2005$, p. 21-34. 
Antrop, M., « The concept of traditional landscapes as a base for landscape evaluation and planning. The example of Flanders Region », Landscape \& Urban Planning, n 38, 1997, p. 105-117.

Baldeschi, P., Dalla razionalità all'identità. La pianificazione territoriale in Italia, Firenze, Alinea, 2002.

Calthorpe, P., Fulton, W., The Regional City, Washington DC, Island Press, 2001.

Corboz, A., « Il territorio come palinsesto », Casabella, n 516, 1985, p. 22-27.

Costanza, R. et al., « The value of the world's ecosystem services and natural capital », Nature, $\mathrm{n}^{\circ}$ 387, 1997.

Dematteis, G., Progetto implicito. Il contributo della geografia umana alle scienze del territorio, Milano, Franco Angeli, 1995.

Di Pietro, G.F., « Il paesaggio come fondamento del Piano territoriale di coordinamento della Provincia di Arezzo », Urbanistica Quaderni, nº 40, 2004.

Donadieu, P., Campagne urbane. Una nuova proposta di paesaggio della città, Roma, Donzelli, 2006 ; Les Campagnes urbaines, Arles, Actes Sud, 1998.

Fanfani, D. (dir.), Pianificare tra città e campagna. Scenari, attori e progetti di nuova ruralità per il territorio di Prato, Firenze, Firenze University Press, 2009.

Gambi, L., « La costruzione dei piani paesistici », Urbanistica, n 85, 1986.

Gambino, R., « Interpretazione strutturale e progetto di territorio », dans Poli, D., (dir.), Il progetto territorialista, numéro monografique de Contesti. Città, territori, progetti, $\mathrm{n}^{\circ} 2,2010$, All'Insegna del giglio, Firenze.

Gambino, R., « Landscape planning. Invarianti e criticità », dans Ronza, M., Mautone, M. (dir.), Patrimonio culturale e paesaggio. Un approccio di filiera per la progettualità territoriale, Roma, Gangemi, 2009, p. 177-180.

Geddes, P., Città in evoluzione, Milano, Il Saggiatore, 1970.

Gisotti, M.R., «L'approche patrimoniale et l'ingénierie territoriale. Deux écoles se confrontent sur un projet territorial », dans Gisotti, M.R. (dir.), Le Projet des parcs agricoles dans les territoires intermédiaires. Cinq scénarios pour la plaine fiorentine, Firenze University Press, 2015.15.renze, 2015.

Gumuchian, H., Pecqueur, B. (dir.), La Ressource territoriale, Paris, Éditions Economica, 2007.

Iacoponi, L., La Bioregione. Verso l' integrazione dei processi socioeconomici e ecosistemici nelle comunità locali, Pisa, ETS, 2001.

Landel, P. A., Senil, N., « Patrimoine et territoire, les nouvelles ressources du développement », dans Développement durable et territoires, dossier 12 «Identités, patrimoines collectifs et développement soutenable ", mis en ligne en janvier 2009, URL : https:// journals.openedition.org/developpementdurable/7563.

Lombardi, L., Giunti, M., Foggi, B., Castelli, C., Santini, G., « Qualità e funzioni ecologiche dei paesaggi : la rete ecologica regionale », dans Marson, A., (dir.), La struttura del paesaggio. Una sperimentazione multidisciplinare per il Piano della Toscana, Roma-Bari, Laterza, 2016.

Maggio, M., Invarianti strutturali nel governo del territorio, Firenze University Press, Firenze, 2014.

Magnaghi, A. (dir.), La pianificazione paesaggistica in Italia. Stato dell'arte e innovazioni, Firenze, Firenze University Press, 2016a. 
Magnaghi, A., « Le invarianti strutturali, fra patrimonio e statuto del territorio », dans Marson, A., (dir.), La struttura del paesaggio. Una sperimentazione multidisciplinare per il Piano della Toscana, Roma-Bari, Laterza, 2016b.

Magnaghi, A., La Biorégion urbaine. Petit traité sur le territoire bien commun, Les Lilas, Eterotopia, 2014.

Magnaghi, A., Il progetto locale, Torino, Bollati Boringhieri, 2000 ; Le Projet local, Liège, Éditions Mardaga, 2003.

Magnaghi, A. (dir.), Il territorio dell'abitare : lo sviluppo locale come alternativa strategica, Milano, Franco Angeli, 1990.

Magnaghi, A., Fanfani, D. (dir.), Patto città, campagna. Un progetto di bioregione urbana per la Toscana centrale, Firenze, Alinea, 2010.

Marson, A. (dir.), La struttura del paesaggio. Una sperimentazione multidisciplinare per il Piano della Toscana, Roma-Bari, Laterza, 2016.

Marson, A., « Esercizi progettuali intorno al territorio inteso come patrimonio », dans Angrilli, M., (dir.), L'urbanistica che cambia. Rischi e valori, Milano, Franco Angeli, 2013.

Millennium Ecosystem Assessment, Ecosystems and Human Well-being : Synthesis, Washington DC, Island Press, 2005.

Mininni, M., Approssimazioni alla città. Urbano, rurale, ecologia, Roma, Donzelli Editore, 2013.

Paba, G., « Militant University : tradizioni e intersezioni nella scuola territorialista », dans Poli, D. (dir.), Il progetto territorialista, numéro monografique de Contesti. Città, territori, progetti, $\mathrm{n}^{\circ} 2,2010$, Firenze, All'Insegna del giglio.

Poli, D., « Il patrimonio territoriale fra capitale e risorsa nei processi di patrimonializzazione proattiva », dans Meloni, B. (dir), Aree interne e progetti d'area, Torino, Rosenberg \& Sellier, 2015.

Poli, D., « Le strutture di lunga durata nei processi di territorializzazione », dans Urbanistica, $\mathrm{n}^{\circ} 147,2011$.

Saragosa, C., L' insediamento umano. Ecologia e sostenibilità, Roma, Donzelli Editore, 2005.

Scazzosi, L., « Limits to transformation in Place's Identity. Theorethical and methodological questions ", dans Roca, Z., Claval P., Agnew, J.A. (dir.), Landscapes, Identities, and Development, Farnham, Ashgate Publishing, 2011, p. 9-24.

Sgard, A., « Le paysage dans l'action publique : du patrimoine au bien commun », Développement durable et territoires, vol. 1, n 2, mis en ligne en septembre 2010, http:// developpementdurable.revues.org/8565.

Thayer, R.L., LifePlace. Bioregional Thought and Practice, Berkeley and Los Angeles, University of California Press, 2003.

TEEB for Business, The Economics of Ecosystems and Biodiversity. Ecological and Economic Foundation, Cambridge, Earthscan, 2012.

Turco, A., Regione e regionalizzazione, Milano, Franco Angeli, 1984.

\section{NOTES}

1. Voir le code des biens culturels et du paysage (D.lgs. $\left.n^{\circ} 42 / 2004\right)$, art. 135 , c. 1. 
2. Selon le texte de la loi 431/85 (connue comme "loi Galasso »), les régions étaient tenues d'élaborer des plans paysagers («piani paesistici o piani urbanistico-territoriali con specifica considerazione dei valori paesistici e ambientali ») relatifs à leur territoire au plus tard le 31 décembre 1986. Cette prescription est restée largement négligée.

3. Convention européenne du paysage, art. 2 [trad. officielle].

4. Voir le code des biens culturels et du paysage (D.lgs. $n^{\circ} 42 / 2004$ ), art. 131, c. 1 [trad. de l'auteur]. Àl'article 1 de la CEP le paysage est défini comme « une partie de territoire telle que perçue par les populations, dont le caractère résulte de l'action de facteurs naturels et/ou humains et de leurs interrelations » [trad. officielle].

5. Gambi écrivait en 1986 : «Quand on parle de territoire on n'évoque pas n'importe quel espace mais un espace défini et déterminé par certains caractères ou, pour mieux dire, par un système de rapports qui unifient ces caractères » (Gambi, 1986, p. 103-104) [trad. de l'auteur].

6. Sur cette interprétation, voir aussi Marc Antrop, qui a défini les paysages traditionnels comme "landscapes having a distinct and recognisable structure which reflects clear relations between the composing elements and having a significance for natural, cultural or aesthetical values.[...] landscapes with a long history, which evolved slowly and where it took centuries to form a characteristic structure reflecting a harmonious integration of abiotic, biotic and cultural elements » (Antrop, 1997, p. 109).

7. Les deux plans ont été approuvés respectivement en février et en mars 2015. Le plan paysager des Pouilles a été coordonné par Alberto Magnaghi (élue de la région des Pouilles: Angela Barbanente) et celui de la Toscane par Paolo Baldeschi (élue de la région Toscane : Anna Marson). Plusieurs plans paysagers actuellement en cours d'élaboration adoptent une interprétation structurelle, évidemment avec des déclinaisons à chaque fois spécifiques (Magnaghi, 2016a).

8. Le livre a été publié en français avec le titre Le Projet local (2003). Toutefois, un texte déjà fondateur de la pensée territorialiste est Il territorio dell'abitare, un ouvrage collectif édité par Magnaghi en 1990.

9. Voir les expériences de l'Ecole d'Empoli, fondée en 2000 au sein de l'université de Florence et aujourd'hui siège des masters en Pianificazione della Città del Territorio e del Paesaggio et en Pianificazione e Progettazione della Città e del Territorio.

10. Afin de faire avancer la vision territoriale et patrimoniale du territoire, Magnaghi et d'autres spécialistes ont fondé en 2011 la Società dei territorialisti, qui regroupe, dans une optique multidisciplinaire, historiens, géographes, architectes-urbanistes, écologues, sociologues, économistes et beaucoup d'autres spécialistes, autour d'un projet de lecture multidisciplinaire du territoire et du paysage.

11. Patrick Geddes écrivait en 1915 : "Chaque lieu a sa personnalité vraie, faite d'éléments uniques, une personnalité qui peut être depuis trop longtemps endormie, mais que l'urbaniste, l'aménageur, l'artiste doit réveiller » (Geddes, 1970, p. 355-356) [trad. de l'auteur].

12. La définition est présente dans le plan paysager de la Toscane et dans la loi régionale toscane 65/2014 « Norme per il governo del territorio », art. 3, c. 1 [trad. de l'auteur].

13. Sur les concepts de patrimoine et de ressource territoriale, voir aussi les interprétations d'Anne Sgard (2010), d'Hervé Gumuchian et Bernard Pecqueur, 2007, de Pierre-Antoine Landel et Nicolas Senil, 2009 et plus en général la réflexion menée par le laboratoire Pacte (Politiques publiques, Action politique, Territoires), unité mixte de recherche CNRS et université de Grenoble. Une synthèse de cette réflexion est contenue dans Daniela Poli, 2015.

14. Les racines culturelles de la biorégion urbaine sont diverses (Saragosa, 2005 ; Paba, 2010). Il existe certainement des liens avec la «coupe de la vallée » et le biorégionalisme de Patrick Geddes, mais aussi avec la « région de la communauté humaine» de Lewis Mumford (The City in History, 1961), et avec les visualisations polycentriques de Benton MacKaye (The new exploration: A philosophy or regional planning, 1928). Dans les années 1960-1980, le terme a été largement utilisé dans la littérature américaine (Peter Berg, Kirkpatrick Sale, Nancy Jack et John Todd, Murray 
Bookchin). Des déclinaisons plus récentes ont été proposées par Calthorpe et Fulton (2001), Iacoponi (2001), Thayer (2003).

15. Ce qui est une évidence, comme on le verra dans les paragraphes suivants, même dans le plan paysager de la Toscane où le diagnostic consacré au territoire rural apparaît prédominant et dont les objectifs de projet développent la préservation de sa multifonctionnalité.

16. La Toscane est une région du Centre-Nord de l'Italie où la persistance d'une robuste tradition civique de gouvernement du territoire se mêle à un paysage très fortement anthropisé, avec des structures historiques bien reconnaissables. Ces caractères représentent peut-être des facteurs à considérer pour comprendre les raisons qui ont conduit à la création de ce plan et le type d'approche adoptée.

17. Regione Toscana 2015, PIT. Piano d'indirizzo territoriale con valenza di piano paesaggistico. Disciplina del piano, art. 6, c. 1) et L.R 65/2014 « Norme per il governo del territorio », art. 3, c. 1 [trad. de l'auteur ].

18. PIT, Disciplina del piano, art. 6, c. 2 [trad. de l'auteur].

19. Cette structure est présente dans les 20 fiches illustrant autant d'unités de paysage régional (dites ambiti). Les fiches sont donc appelées schede d'ambito.

20. PIT, Disciplina del piano, art. 6, c. 3 [trad. de l'auteur].

21. Voir PIT, Abachi des invariantes structurelles.

22. Voir, à ce propos, le travail présenté dans la partie relative à la $2^{\mathrm{e}}$ Invariante structurelle du plan, « Les caractères éco-systémiques » (voir aussi Lombardi et al., 2016).

23. Sur ce sujet voir aussi le plan paysager des Pouilles.

24. À cette fin, le plan Toscane réalise des dispositions combinées avec la L.R. 65/2014 qui introduit une distinction entre territoire rural et territoire urbanisé, le seul où de nouvelles constructions sont encore autorisées. L'outil permettant la mise en œuvre de cette disposition juridique est la carte du territoire urbanisé, contenue dans la section relative à la $3^{\mathrm{e}}$ Invariante structurelle du plan paysager.

25. Fiche de l'ambito 06 Firenze-Prato-Pistoia, p. 68 [trad. de l'auteur].

26. Toujours sur ce thème, voir aussi le document du plan paysager Linee guida per la riqualificazione paesaggistica del margine dei tessuti urbani della città contemporanea.

\section{RÉSUMÉS}

L'article propose une réflexion sur l'approche territorialiste de la planification du paysage en Italie. Le fondement de cette posture scientifique est le concept de patrimoine territorial et paysager, organisme complexe aux valeurs culturelles et en même temps fonctionnelles. L'article illustre les paradigmes principaux de l'approche patrimoniale et son application dans le plan paysager de la Toscane. À partir de l'étude de cas, la conclusion suggère des pistes de réflexion pour la mise en œuvre de tels instruments, qui visent le passage d'une préservation statique du paysage à une préservation dynamique.

The article offers a reflection on the territory-based approach to landscape planning in Italy. The notion upon which this scientific approach is based is that of territorial and landscape heritage, a complex set of cultural and functional values. The article describes the principal paradigms in the heritage approach and the way it is applied in the Tuscan landscape plan. Based on a case 
study, the article concludes by suggesting further ideas for deploying such instruments aimed at shifting from a static to a dynamic preservation of the landscape.

INDEX

Keywords : landscape plan, territorial heritage, Italy, co-evolution, territorial project Mots-clés : plan paysager, patrimoine territorial, Italie, coévolution, projet de territoire

\section{AUTEUR}

\section{MARIA RITA GISOTTI}

Maria Rita Gisotti, architecte-urbaniste, $\mathrm{PhD}$ en projet urbain et territorial, est maître de conférences à l'École d'architecture de l'université de Florence. Ses domaines de recherche sont l'approche patrimoniale, l'analyse et l'aménagement du paysage à l'échelle territoriale et régionale, le rapport ville-campagne et les territoires périurbains.

mariarita.gisotti[at]unifi[dot]it 